\title{
A CLASSIFICATION OF MODULES OVER COMPLETE DISCRETE VALUATION RINGS
}

\author{
BY CHANG MO BANG ${ }^{1}$
}

Communicated by Richard Pierce, October 17, 1969

1. Introduction. The purpose of this paper is to announce the completion of a classification (up to isomorphism) of all modules which are direct sums of countably generated modules over complete discrete valuation rings. The detailed proofs will appear elsewhere. Throughout this paper, let $R$ denote a fixed but arbitrary complete discrete valuation ring and $p$ a fixed but arbitrary prime element of $R$. For the sake of convenience, a cardinal is viewed as the first ordinal having that cardinality. Let $(c, R, k)$ be the class of all countably generated reduced $R$-modules of (torsion-free) rank $\leqq k$ and $D(c, R, k)$ that of all direct sums of members of $(c, R, k)$. Clearly

$$
\begin{array}{ccc}
(c, R, 0) & \subset(c, R, 1) & \subset \cdots \subset(c, R, \omega) \\
\cap & \cap & \\
D(c, R, 0) & \subset D(c, R, 1) \subset \cdots \subset D(c, R, \omega) .
\end{array}
$$

Notice that a $p$-primary abelian group is a member of $(c, R, 0)$, particularly if $R$ is a ring of $p$-adic integers. A classification (of all members) of $(c, R, k)$ was done by Ulm (1933) when $k=0$ [8], by Kaplansky and Mackey (1951) when $k=1$ [4], by Rotman and Yen (1961) when $k<\omega[7]$, and that of $D(c, R, k)$ was done by Kolettis (1960) when $k=0$ [5]. First, we complete a classification of $(c, R, \omega)$ and then, utilizing this, we finish that of $D(c, R, \omega)$.

2. Invariants. We need two kinds of invariants, namely, the Ulm invariants and the basis types. Since the celebrated Ulm invariants are well known, a brief explanation of the basis types only is in order [2], [4], [7]. Let $R^{k}=\oplus\{R: i<k\}$ for each $k$. Define $f(R)$ to be the class of all sordinal (ordinal or $\infty$ ) valued functions on $R^{k}$ for all cardinals $k$, and $m(Q)$ that of all square row-finite matrices over $Q$, the quotient field of $R$. Suppose that $f, g \in f(R)$. Define $f \sim g$ to mean

AMS Subject Classifications. Primary 2030, 2027; Secondary 1340, 1350.

Key Words and Phrases. Countably generated module, complete discrete valuation ring, classification up to isomorphism, torsion-free rank, Ulm invariant, reduced module, $p$-adic integers, basis, basic free submodule, basis type, summandwise basis, height-preserving isomorphism, $p$-height.

1 The author was supported in part by NSF Grant GP-8725 and GP-11804. 
both that Dom $f=\operatorname{Dom} g=R^{k}$ for some cardinal $k$ and that there is a matrix $\gamma$ and a diagonal matrix $\delta$, both $k \times k$ invertible integral (that is, all entries are elements of $R$ ) in $m(Q)$, such that $f(\alpha \gamma)=g(\alpha \delta)$ for all $\alpha \in R^{k}$. It is routine to show that $\sim$ is an equivalence relation on $f(R)$.

Let $M$ be an $R$-module of rank $k$. Then, every basis $\eta=\left\{y_{i}: i<k\right\}$ defines a function $g$ of $f(R)$ by

$$
g(\alpha)=h_{p}(\alpha \eta)=h_{p}\left(\sum\left\{a_{i} y_{i}: i<k\right\}\right)
$$

for all $\alpha=\left\{a_{i}: i<k\right\} \in R^{k}$. Notice that $g=\infty$ if $k=0$ since a sum without term is 0 . It is routine to show that $g \sim g^{\prime}$ if $g^{\prime}$ is defined by another basis of $M$. Thus, $M$ determines uniquely a class of $f(R) / \sim$, which we call the basis type of $M$. It is easy to show the following lemma.

Lemma 1. Two reduced $R$-modules $M$ and $M^{\prime}$ have the same basis type if and only if they contain basic free submodules $F$ and $F^{\prime}$, respectively, with a height-preserving isomorphism from $F$ onto $F^{\prime}$.

3. A classification of $(c, R, \omega)$.

TheOREM 1. Let $M$ and $M^{\prime}$ be countably generated reduced $R$-modules. Then, $M \simeq M^{\prime}$ if and only if they have the same Ulm invariant and the same basis type.

Only the "if" part needs a proof. Let $\alpha=\left\{a_{i}: i<k\right\}$. Define $\alpha(r)$ $=\left\{a_{i}: i<r\right\}$ for each number $r$. Let $k$ be the same rank of $M$ and $M^{\prime}$. Then, by Lemma 1 , there are ordered bases $\eta=\left\{y_{i}: i<k\right\}$ and $\eta^{\prime}=\left\{y_{i}^{\prime}: i<k\right\}$ of $M$ and $M^{\prime}$, respectively, with a height-preserving isomorphism $\rho$ such that $\rho(\alpha \eta)=\alpha \eta^{\prime}$ for all $\alpha \in R^{k}$. We may assume that there are countable subsets $\xi=\left\{x_{i}: i<\omega\right\}$ and $\xi^{\prime}=\left\{x_{i}^{\prime}: i<\omega\right\}$ of $M$ and $M^{\prime}$, respectively, such that

$$
\begin{aligned}
& M=[\xi \cup \eta] \text { and } M^{\prime}=\left[\xi^{\prime} \cup \eta^{\prime}\right] \quad \text { with } \\
& p x_{i} \in[\xi(i) \cup \eta(i)] \text { and } p x_{i}^{\prime} \in\left[\xi^{\prime}(i) \cup \eta^{\prime}(i)\right] \quad \text { for each } i<\omega .
\end{aligned}
$$

The main idea of the proof is to construct a sequence of heightpreserving isomorphisms $\left\{\phi_{i}: i<\omega\right\}$ in such a way that the following conditions are satisfied.

(a) $\left.\phi_{i}: A_{i}\right\rangle \rightarrow A_{i}^{\prime}$ where

$$
\begin{aligned}
A_{i} & =\left[\xi(i) \cup \cup_{\eta}(i) \cup \phi_{i}^{-1}\left(\xi^{\prime}(i) \cup \eta^{\prime}(i)\right)\right], \\
A_{i}^{\prime} & =\left[\xi^{\prime}(i) \cup \eta^{\prime}(i) \cup \phi_{i}(\xi(i) \cup \cup(i))\right] .
\end{aligned}
$$

(b) $\phi_{0} \leqq \cdots \leqq \phi_{i} \leqq \phi_{i+1} \leqq \cdots \cdot$

(c) There exists a nonnegative integer $n(i)$ such that $p^{n(i)} A_{i}$ 
$\subseteq[\eta(i)]$ and $p^{n(i)} A_{i}^{\prime}=\left[\eta^{\prime}(i)\right]$ and $\phi_{i}=\rho$ as height-preserving isomorphism from $p^{n(i)} A_{i}$ onto $p^{n(i)} A_{i}^{\prime}$.

The supremum of $\left\{\phi_{i}: i<\omega\right\}$ gives the required isomorphism from $M$ onto $M^{\prime}$. For more detailed proof, see [1] or [2].

4. A classification of $D(c, R, \omega)$.

TheOREM 2. Let $M$ and $M^{\prime}$ be direct sums of countably generated reduced $R$-modules. Then, $M \simeq M^{\prime}$ if and only if they have the same Ulm invariant and the same basis type.

Again, only the "if" part needs a proof. We may write as

$$
M=\oplus\left\{M_{i}: i \in I\right\} \text { and } M^{\prime}=\oplus\left\{M_{i}^{\prime}: i \in I\right\}
$$

where all $M_{i}, M_{i}^{\prime} \in(c, R, \omega)$ and $I$ is a cardinal. For notational convenience, define $M(T)=\oplus\left\{M_{i}: i \in T\right\}, T \subseteq I$. The main idea of the proof is to show that there is a partition of $I$ into countable subsets $\left\{I_{j}: j<I\right\}$ such that, for each $j<I, M\left(I_{j}\right)$ and $M^{\prime}\left(I_{j}\right)$ have the same Ulm invariant and the same basis type. Then by Theorem 1, they are isomorphic and, consequently, $M \simeq M^{\prime}$. In fact, by the Kolettis theorem [3], [5], [6], we may assume that $M_{i}$ and $M_{i}^{\prime}$ have already the same Ulm invariant for each $i$. The following lemmas indicate the route of the proof.

Lemma 2. Let $N=A \oplus B \oplus C$ be a reduced $R$-module such that the following conditions are satisfied.

(a) There are in $N$ disjoint subsets $\eta_{A}$ and $\eta_{B}$ such that $\eta_{A}$ and $\eta_{A} \cup \eta_{B}$ are bases of $A$ and $A \oplus B$, respectively.

(b) If $x_{A} \in\left[\eta_{A}\right]$ and $x_{B} \in\left[\eta_{B}\right]$, then ( $h_{N}$ denotes the p-height in $N$ )

$$
h_{N}\left(x_{A}+x_{B}\right)=\min \left\{h_{N}\left(x_{A}\right), h_{N}\left(x_{B}\right)\right\} .
$$

Then, if we write $\eta_{B}=\left\{y_{i}: i<k\right\}, k=\left|\eta_{B}\right|$, there is in $m(Q)$ a $k \times k$ diagonal invertible integral matrix $\delta=\left\{d_{i}: i<k\right\}$ such that the following conditions are satisfied.

(c) $\tau=\Pi_{B}\left(\delta \eta_{B}\right)$ is an ordered basis of $B$. (Here, $\Pi_{B}$ is the canonical projection of $N$ onto $B$.)

(d) There is a height-preserving isomorphism $\rho$ from $\left[\delta \eta_{B}\right]$ onto $[\tau]$ such that $\rho\left(\alpha \delta \eta_{B}\right)=\alpha \tau$ for all $\alpha \in R^{k}$.

Lemma 3. Let $k$ be the rank of $M$ and $M^{\prime}$. Let $\eta=\left\{y_{i}: i<k\right\}$ and $\eta^{\prime}=\left\{y_{i}^{\prime}: i<k\right\}$ be ordered bases of $M$ and $M^{\prime}$, respectively, with $\eta^{\prime}$ summandwise (ihat is, each $y_{i}^{\prime} \in M_{j}$ for a $j$ ). If $J$ is a countable subset of $I$, then there is a set $T$ such that the following conditions are satisfied.

(a) $T$ is countable and $J \subseteq T \subseteq I$. 
(b) Define $\eta(T)=\left\{y_{i} \in \eta: y_{i} \in M(T)\right\} . \eta(T)$ and $\eta^{\prime}(T)$ are bases of $M(T)$ and $M^{\prime}(T)$, respectively.

(c) $y_{i} \in \eta(T)$ if and only if $y_{i}^{\prime} \in \eta^{\prime}(T)$.

Lemma 4. $M$ and $M^{\prime}$ have the same basis type if and only if there is a partition of $I$ into countable subsets $\left\{I_{j}: j<I\right\}$ such that $M\left(I_{j}\right)$ and $M^{\prime}\left(I_{j}\right)$ have the same basis type for each index $j<I$.

Using Lemma 2, 3 and a transfinite induction, we can prove Lemma 4. Theorem 2 is immediate from Lemma 4.

CoROLlARY. $M \simeq M^{\prime}$ if and only if they have isomorphic torsion parts and contain basic free submodules $F$ and $F^{\prime}$ of, respectively, with a height-preserving isomorphism from $F$ onto $F^{\prime}$.

The author would like to acknowledge his gratitude to Professor Charles K. Megibben for suggesting the problems solved in this paper and for his invaluable guidance.

\section{BIBLIOGRAPHY}

1. C. Bang, Countably generated modules over complete discrete valuation rings, J• Algebra (to appear).

2. - Modules over complete discrete valuation rings, Dissertation, Vanderbilt University, Nashville, Tenn., 1969.

3. P. Hill, Sums of countable primary groups, Proc. Amer. Math. Soc. 17 (1966), 1469-1470. MR 33 \#7408.

4. I. Kaplansky and G. Mackey, A generalization of Ulm's theorem, Summa Brasil. Math. 2 (1951), 195-202. MR 14, 128.

5. G. Kolettis, Jr., Direct sums of countable groups, Duke Math. J. 27 (1960), 111-125.

6. F. Richman and E. Walker, Extending Ulm's theorem without group theory, Proc. Amer. Math. Soc. 21 (1969), 194-196.

7. J. Rotman and T. Yen, Modules over a complete discrete valuation ring, Trans. Amer. Math. Soc. 98 (1961), 242-254. MR 23 \#A227.

8. H. Ulm, Zur Theorie der abzahlbar-unendlichen Abelschen Gruppen, Math. Ann. 107 (1933), 774-803.

Emory University, Atlanta, Georgia 30322 and

VANDERbilt University, Nashville, TenNessee 37203. 